\title{
INDUSTRIAL APPLICATION OF MACHINE VISION
}

\author{
S.Sathiyamoorthy ${ }^{1}$ \\ ${ }^{1}$ Project Scientist IV, NHHID, Anna University, Tamil Nadu, India
}

\begin{abstract}
Inspection of components using machine vision technologies provides solution for quality and process control. Various applications of Machine vision technologies are automotive, Pharmaceutical, food and beverage, electronics, packages, process control and special application. In this paper dimensional measurement, optical character reorganization, process control using image processing, checking of presence and absence of finished product parts in the production line are discussed. The basic idea of this paper is to make aware of machine vision technology and to improve the production quality, reduce the scrap product due to non-conformity by controlling the manufacturing process through machine vision and also to prevent the value addition for scrap product in the subsequent stage of manufacturing process. Various projects discussed in this paper are implemented and proofs of concepts are shown to the manufacturer.
\end{abstract}

Keywords: Optical gauging/Video measurement, Barcode and Optical character recognition(OCR) reading, process control, Bottle level inspection and Blister pack inspection..

\section{INTRODUCTION}

(MV) is the knowledge and approaches used to provide image-based automatic examination for quality control, process control, and robot guidance. Basically machine vision is used for optical gauging, quality assurance, sorting, part assembly inspection, presence and absence and controlling the manufacturing process.

\section{Operation of Machine Vision}

MV system operation starts with acquisition of an image using appropriate cameras, lenses and light depending on the application.

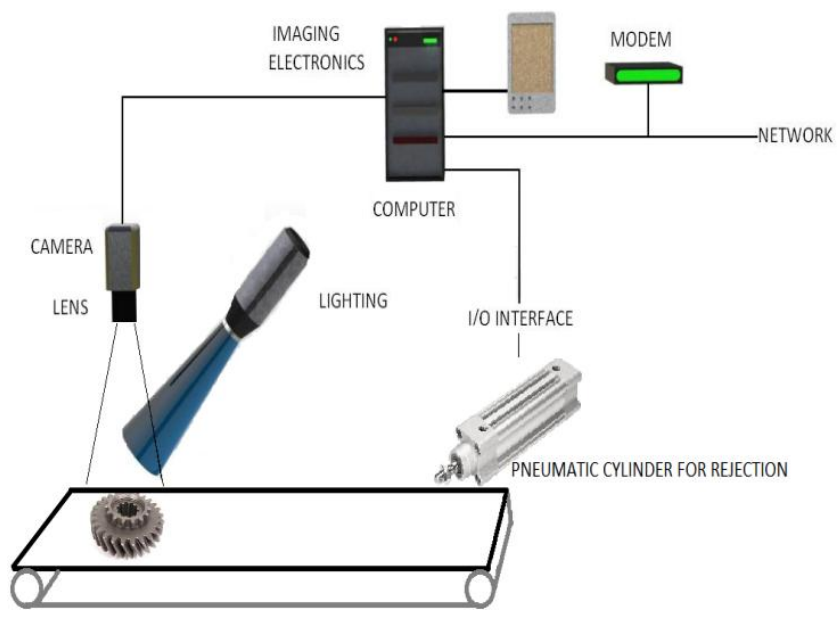

Fig -1: Machine Vision Concept.

LabVIEW, MV Impact, OpenCV, MATLAB, these software's will provide various digital image processing technique used for obtaining required information from an acquired image. Based on the extracted image the processor will take decision i.e. acceptable or Not acceptable. Thus
NOT acceptable component will be removed from the production line using ejection mechanism such as actuator or blower.

2

\section{MEASUREMENT OF FAN SHAFT}

In fan shaft there are two important areas are to be measured perfectly, where two bearings are inserted in the shaft. If the fan shaft diameter is more, it is not possible to insert the shaft into the standard sized bearing. So before insertion of bearing in shaft diameter it is ensured using machine vision [6]. In this, the requirement is to detect the bearing seating diameters located in two places of each shaft with the below classifications:

i) OK component.

ii) Not OK component - over size.

iii) Not OK component - under size.

Over size components is goes to rework, under size component components goes as scrap. For a given model, the operator selects the model from the monitor. The object is placed on the holder. The operator then presses the leg pedal which provides a trigger to the light and the camera. Then the cameras capture the image and transferred to the PC. The images are processed[2] by the software LabVIEW and the output is displayed on the monitor.

Algorithm used: Edge Detector. The algorithm was developed to count the number of pixel along the edge of the shaft, by just counting the number of pixels which falls into the range gives acceptance level of the shaft. Then the pixel value is also calibrated into real world.

Due to back light effect the object silhouette is taken as the image. In that Sudden changes in the grey scale value forms the edges by calculating the number of pixels that covers the 
two edges in one axis forms the measurements of the object , then the pixels values are calibrated and converted into real time value.

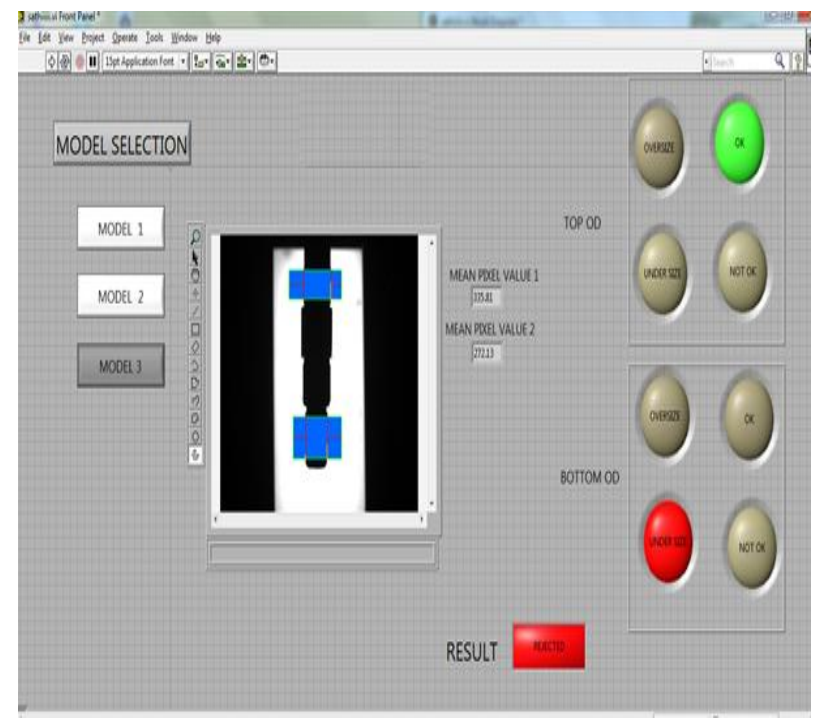

Fig -2: Front Panel for fan shaft diameter inspection

\section{BARCODE AND OCR READING}

\subsection{Engine Assembly}

Problem Description: There are many types of engine such as $350 \mathrm{cc}, 500 \mathrm{cc}$, and Import and export model. By keeping barcode and model number as a reference operators assemble the corresponding tappet door which indicates the model name as Optical Character Reorganization., by tacking/seeing this tapper door OCR as a reference the subsequent operators in the next stage will assemble the parts to that engine. There may be a possibility of keeping wrong tapper OCR on the engine block, because of different models operator may be confused, this will lead to wrong assembling of parts in the subsequent stage.

In order to control this process of engine assembly MV system is used. MV System capture the image of barcode and OCR, barcode gives the information about parts to be assembled and OCR gives information about engine model number. Then these images are processed and checks with the corresponding part are assembled or not. If it is OK engine will be passed to next station otherwise engine is blocked using locking piston actuator and graphical user interface show which part have to assemble. GUI gives database information of how many Ok and how many NOT OK components inspected.

\subsection{Description of System Operation}

The Component which is to be inspected is placed on the inspection table. The presence of component is sensed by the proximity sensor and it triggers the actuators to lock the engine crank case and vertical and horizontal pneumatic actuators. Once the actuators comes to the retarded position the cameras are triggered for taking the required images. After taking the images, the Cameras are sent back to their respective home positions by the actuators which are again controlled by another set of reed sensors. From the observed images the compatibility between images are checked. Following image Fig.3. shows the user interface of the inspection system. In case the codes are similar, the same is indicated through green label.

In case of dissimilarity, a red label is indicated as shown in figure 4. Once the red label indication is observed in the display, simultaneously the interlock system will disable the air supply of the impact screw gun, thereby suspending further operation on the unit. Once the appropriate tappet cover is replaced, then reset button is pressed to continue the inspection cycle. In addition, there will be a master stop button to stop the operation in case of any eventuality or emergency.

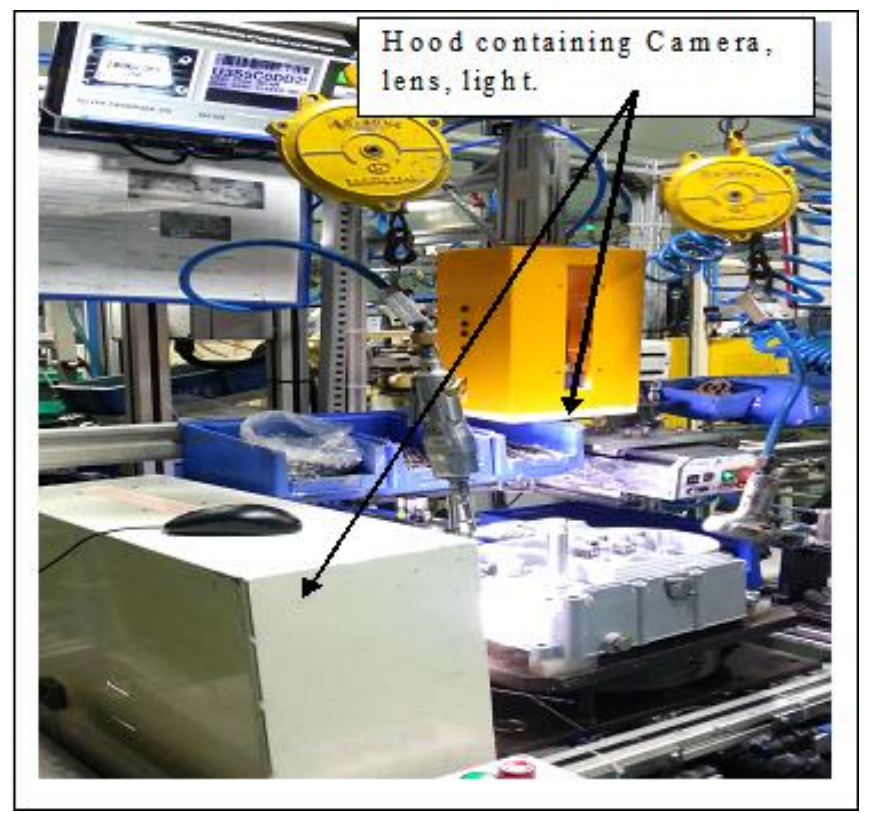

Fig -3: Machine vision setup for Barcode and OCR Reading.

The inspection system is integrated with existing frame set up. Model of the same is shown below as Figure 2.

Algorithm Used: Thresholding and Segmentation, Particle filtering, morphological techniques, coordinate reference system, OCR toolbox in LabVIEW.

Automation: PLC program for the sequence of automatic operation. PLC is used to control the pneumatic operation camera and light triggering for capturing the images. Fig -4: Front Panel for OCR and Barcode Inspection. If wrong component is placed by an operator, a popup will be appeared in the front Panel showing the picture of components to be placed, based on this information operator remove wrong component and places correct component. 


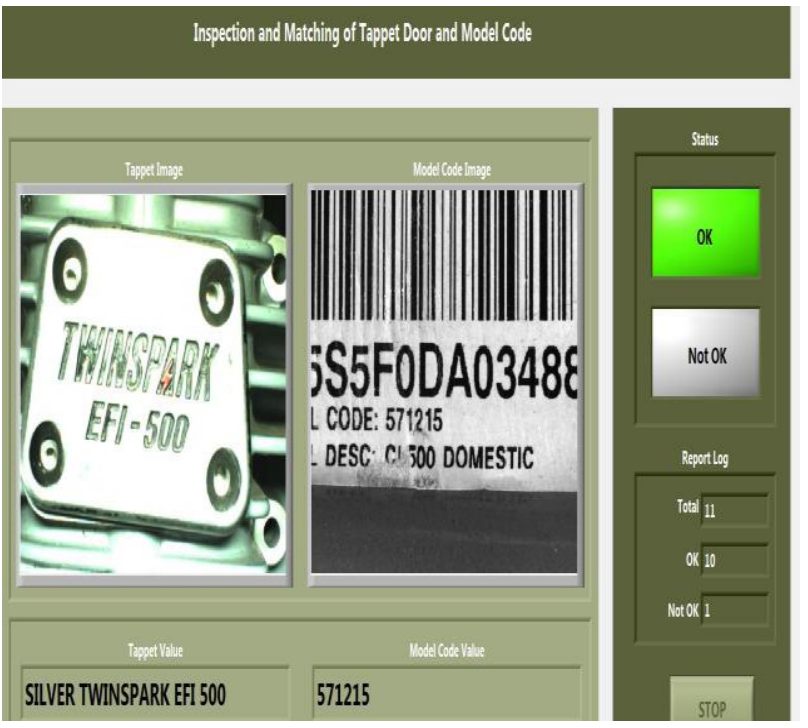

Fig -4: Front Panel for OCR and Barcode Inspection

\section{PROCESS CONTROL IN MANUFACTURING}

\subsection{Mechanical Punch Press}

Automobile components are manufactured using mechanical punch press (flywheel type). The shape of the auto components is determined by DIE. During the punching process sometimes the components may sticks on to the top die. The operator not able to see the top die and operator places another material and presses the pedal. This action leads to double punch and damages the dies. If die is damaged and it is not noticed then the components produced after die dame will not meets the finished product requirement such as outer and inner diameter so, the manufactured components will go as scrap. Replacing the damaged die with new die is costlier.

To control this process a camera is placed in such a way to see the upper die. Then the camera is triggered by placing the limit switch in the pedal for capturing the image.

If the component is attached in the top die pneumatic actuator is used to lock the pedal. So the operator is not able to operate the machine for the next time if the component is attached on the top die. In this way the manufacturing process is controlled.

The machine vision system will have a camera, a light and PC. In addition, there will be an actuator to arrest the movement of the leg pedal in case of any fault. The sequence of operation is explained below:

a) The operator places the component and presses the pedal

b) When the pedal is pressed down for releasing the top DIE to be pressed down, at the end of the stroke, it activates an electrical switch placed in the bottom of pedal. This triggers the camera and the light and takes the images of the top surface of the die. c) In case the object is struck on top, the processor gives signal to camera further camera activates a pneumatic actuator which will block the pedal moving downwards[5]. In addition, a red lamp and an alarm can also be initiated to alert the operator.

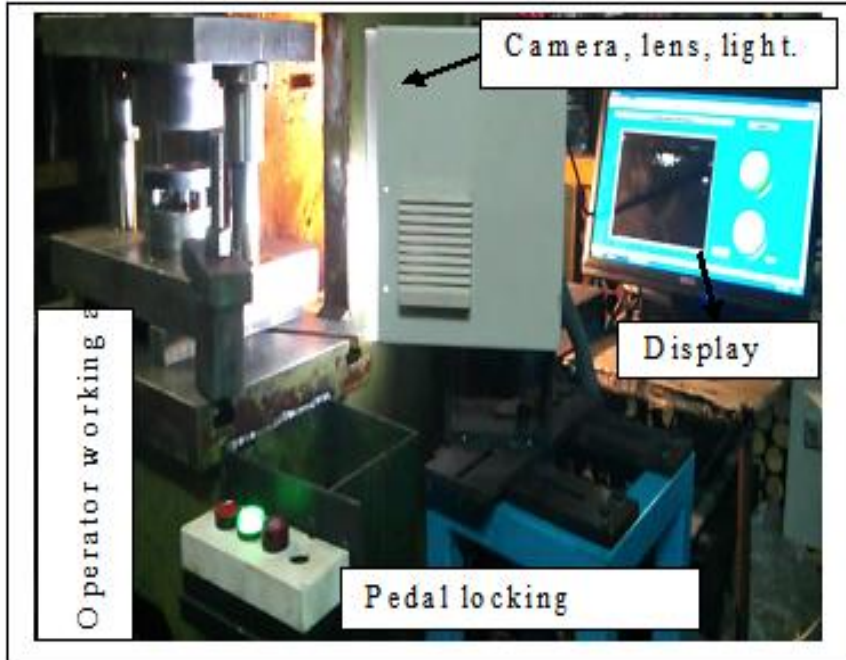

Fig - 5: Machine Vision setup for controlling Die damage.

Algorithm Used: Grayscale image to binary image by Thresholding and segmentation, advance morphology, particle analysis (area), Particle Filter, masking.

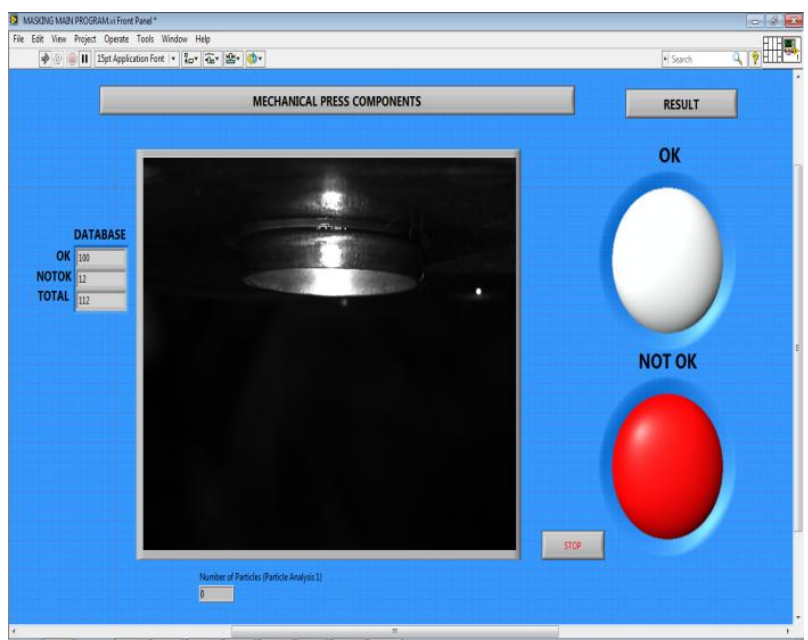

Fig -6: Front Panel for Controlling Die Damage

\section{PHARMACEUTICAL APPLICATION}

\subsection{Presence and Absence of Bottle Cap and Level Inspection}

Level of medicine in vial, glucose bottle, tonic bottle is ensured by Machine vision system. If the level is not good it is removed in the production line itself. Fig -7 demo setup for real time Automatic bottle level, cap presence and absence inspection 


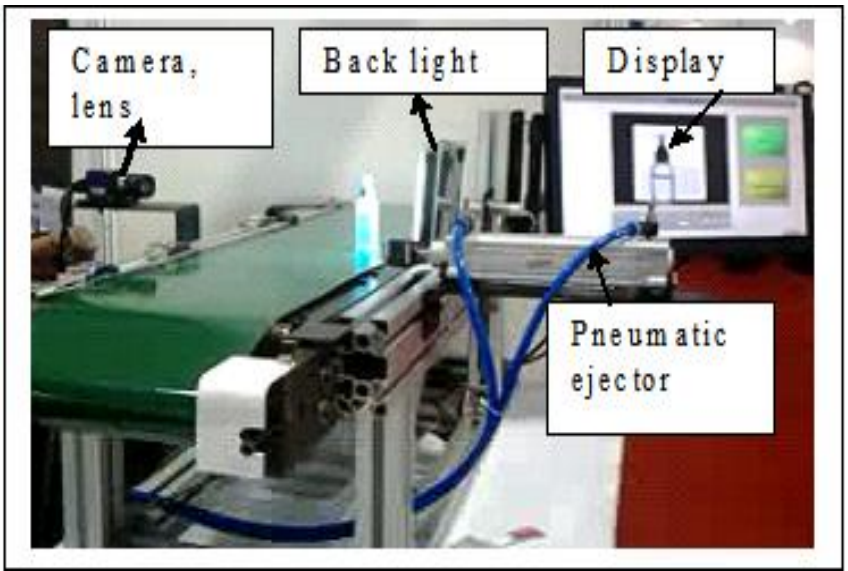

Fig -7: Machine Vision set up for Automatic bottle level, cap presence and absence inspection

Bottles to be inspected will be moving in the conveyer, the presence of the bottle is sensed by proximity sensor which will trigger the camera and the image is captured and send it PC via gigabit Ethernet cable where it checks with algorithms created in LabVIEW and takes the decision that the bottles meets the requirement or not if it meets requirements such as level and presence of cap, then the bottles gores to $\mathrm{OK}$ bin otherwise the pneumatic actuator will eject NOTOK components to NOT OK bin.

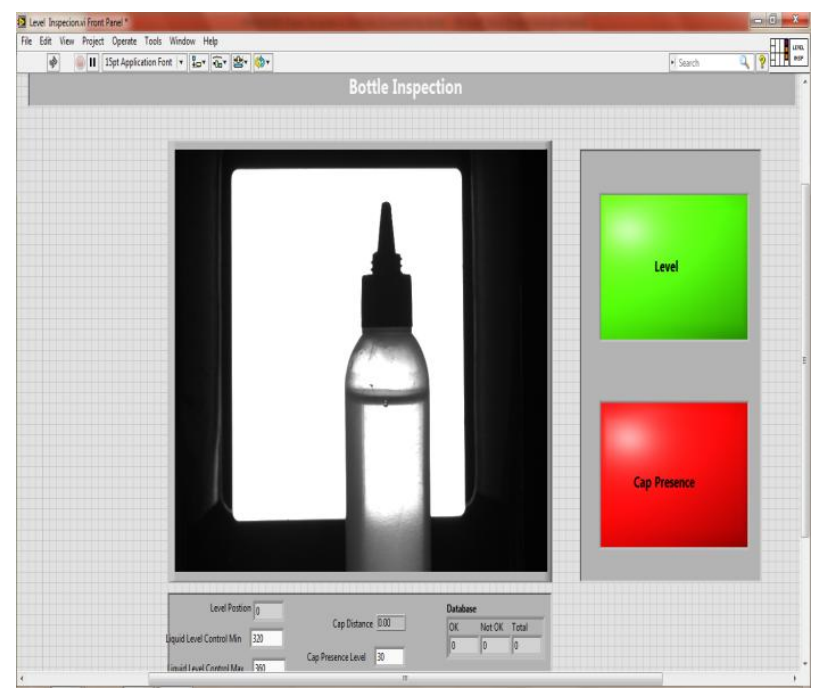

Fig -8: Front Panel for Level, cap presence and Absence Inspection

Algorithm Used: Edge detector [10], the algorithm was developed to count the number of pixel along the edge of the bottle, by just counting the number which falls into the range give acceptance level and cap presence or absence

\subsection{Blister Pack Inspection}

Blister pack inspection for its completeness is very important. Patient may think tablet is available in the blister pack in case of any emergency conditions occurs if there is no tablets in the packed or wrapped blister pack it would become a big problem at the customer end. It is necessary to inspect the following components in the blister pack.
a) Missing Tablets.
b) Broken Tablets.
c) Half tablets.
d) Color Sequence Verification.
e) Label Reading.

If any of the above parameters is failed, then the pneumatic blower is used to remove the blister pack in the production line itself.

MV system containing a Camera, a doom light, pneumatic ejector and sensor are mounted on the conveyor. As the blister packs moves over conveyor, sensor senses its presence, triggers the light and the camera. As the blister is covered with white top, a special dome light is used to provide the proper image. Images are taken, processed in a PC and the output is displayed on the monitor. Image in fig.9 shows the processed output. At the end of inspection, OK blister packs moves itself onto $\mathrm{OK}$ bin at the end of conveyor. In case of NOT OK blister pack, it is pushed into NOT OK bin by an air blower nozzle.

In addition with that data base also generated which includes total components included, number of $\mathrm{OK}$ components inspected and number of not ok components inspected. Based on this corrective action will be done and used for stock verification.

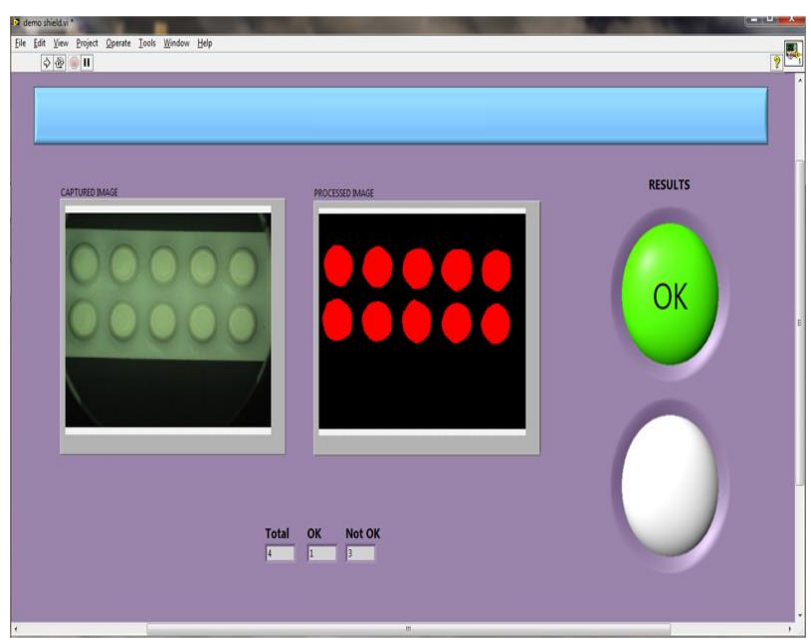

Fig -9: Front panel for Blister pack Inspection-showing OK pack

Algorithm used: Color plane extraction HSL hue, saturation \& light Plane, Thresholding (grey to binary), filter (smoothing-local averaging), and advance morphology, Particle analysis and particle filter.

Automation: Automation is done using Omron PLC, by getting the NOT OK signal from processor pneumatic blower is activated using PLC. 


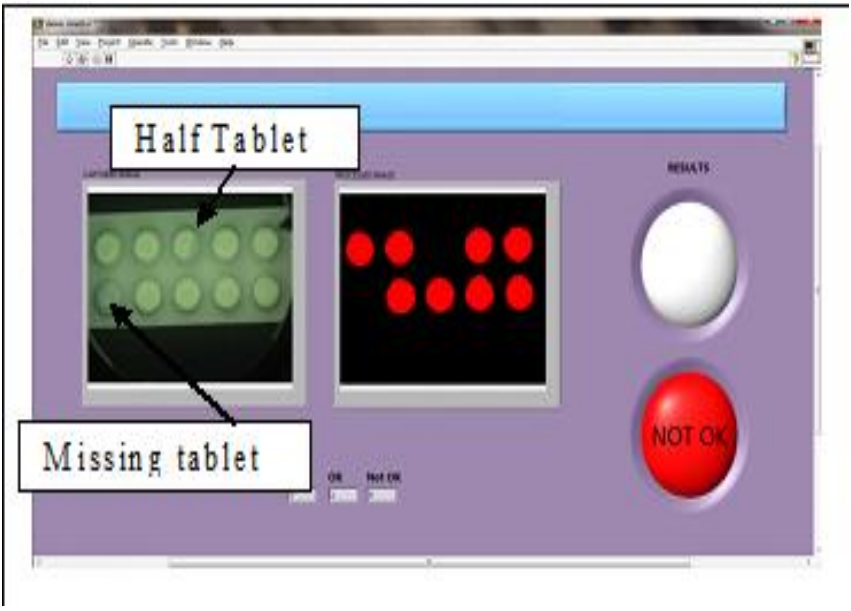

Fig -10: Front Panel for Blister Pack Inspection-showing Missing and half tablets.

\section{CONCLUSIONS}

The aim this paper is to make aware of machine vision technologies which are applicable to various industries. to improve the production quality, reduce the scrap product due to non-conformity by controlling the manufacturing process through machine vision and also to prevent the value addition for scrap product in the subsequent stage of manufacturing process

\section{ACKNOWLEDGMENTS}

As I have worked as a Project Engineer, Various projects discussed in this paper are implemented and proofs of concept were demonstrated in various industries. This work was Supported by TIFAC CORE In Machine vision and Nanchil Technologies Pvt Ltd. Chennai.

\section{REFERENCES}

[1] Golnabi H, Asadpour A.: Design and application of industrial machine vision systems. Robotics and Computer-Integrated Manufacturing, 23(2007), 630637.

[2] Zhang Z., Chen Z., Shi J., Jia F., Dai M.: Surface roughness vision measurement in different ambient light conditions. $15^{\text {th }}$ Int. Conf. Mechatronics and Machine Vision in Practice, Auckland (New Zealand), 2-8 December 2008.

[3] Bhuvanesh A., Ratnam M.: Automatic detection of stamping defects in lead frames using machine vision: overcoming translational and rotational misalignment. Int. J. Adv. Manuf. Technology, 32 (2007), pp 1201-1210.

[4] Remigiusz Labudzki, Stanislaw Legutko : Applications of Machine Vision

[5] Cheng Y., Jafari M.: Vision-based online process control in manufacturing applications. Trans. on Automation science and engineering, 5(2008)1, pp 140-153.

[6] Jian-hai H., Shu-shang Z., Wei S.: Research on sub pixel detecting on-line system based on machine vision for inner diameter of bearings. Int. Conf. on Robotics and Biometics, Sanya, 15-18 December 2007, pp 2049-2052

[7] Fernandes, A.O.; Moreira, L. F E; Mata, J.M., "Machine vision applications and development aspects," Control and Automation (ICCA), 2011 9th IEEE International Conference on , vol., no., pp.1274,1278, 19-21 Dec. 201

[8] Swapna, P.; Krouglicof, N.; Gosine, R.G., "The question of accuracy with geometric camera calibration," Electrical and Computer Engineering, 2009. CCECE '09. Canadian Conference on , vol., no., pp.541,546, 3-6 May 2009

[9] Hsu, R.C.; Ping-Wen Kao; Wei-Jie Lai; Cheng-Ting Liu, "An initial edge point selection and segmental contour following for object contour extraction," Control Automation Robotics \& Vision (ICARCV), 2010

\section{BIOGRAPHIES}

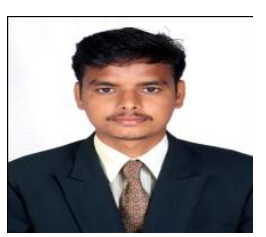

S. Sathiyamoorthy, currently working as a Project Scientist IV, National Hub for Healthcare Instrumentation Development, Anna University, Chennai Completed M.E -Control and Instrumentation, B.E- Electronics and Communication Research Areas: Machine Vision-Digital Image Processing, Calibration of Instruments, Indigenous Instrumentation development, Hybrid Energy, Sensors and automation.

E-mail: sathiyamano@gmail.com 